\title{
Robot Assisted Thoracic Surgery (RATS)
}

\author{
Naohiro Kajiwara, Masatoshi Kakihana, Jitsuo Usuda, Tatsuo Ohira, \\ Norihiko Kawate and Norihiko Ikeda
}

Additional information is available at the end of the chapter

http://dx.doi.org/10.5772/53896

\section{Introduction}

Robotic surgery using the da Vinci ${ }^{\circledR}$ Surgical System (dVS; Intuitive Surgical, Inc., Sunnyvale, CA, U.S.A) has been approved in many countries for cardiovascular surgery, urology, and gynecology. However, unlike mediastinal tumors, which can be located in many different sites, in other organs treated with the dVS (heart, bladder, prostate, and uterus), the targets are generally found in similar locations. Moreover, the anatomical structures surrounding mediastinal tumors are vital (heart, aorta, lungs, pulmonary vessels, esophagus, and vertebrae), and it is crucial to pay careful attention in performing operations. Video-assisted thoracic surgery (VATS) is less invasive than standard thoracotomy, but there is the problem of postoperative pain caused by the inevitable leverage of instruments on the chest wall during the procedure, and the difficulty of manipulation in the mediastinum. The most crucial aspect is the limited movement of long rigid instruments (stick surgery). The instrument arms used in the dVS inflict less stress on the chest wall than conventional VATS, especially when highly skilled techniques are needed to operate on lesions in difficult-to-access areas [1].

Recently, Ruckert and his colleagues reported a comparison of robotic and nonrobotic thoracoscopic thymectomy [2]. After a follow-up of 42 months, they reported the cumulative complete remission rates of myasthenia gravis for robotic and nonrobotic thymectomy were $39 \%$ and $20 \%$, respectively. Their conclusions reveal an improved outcome for myasthenia gravis after robotic thoracoscopic thymectomy compared with thoracoscopic thymectomy. In November 2011, Park and his colleagues reported robotic lobectomy for non-small cell lung cancer (NSCLC) concern with long-term oncologic results [3]. This report is the first concerning outcome about long term prognosis of lung cancer the robotic surgery in a total of 325 consecutive patients underwent robotic lobectomy for early-stage NSCLC at 3 institutions. They reported that overall 5-year survival was $80 \%$ and by pathologic stage, $91 \%$ for stage IA, $88 \%$ for stage IB and $49 \%$ for all patients with stage II disease. They 
concluded robotic lobectomy for early-stage NSCLC can be performed with low morbidity and mortality and long-term stage-specific survival is acceptable and consistent with previous results for VATS and thoracotomy. These data are very important, and these two reports support the scientific basis of robotic surgery. Thus, robotic surgery has gradually produced improved clinical outcomes, making it attractive for many surgeons. In the near future, robotic surgery may replace conventional VATS.

In this chapter, we set out to establish a procedure for resection of a variety of targets located in various thoracic areas difficult to reach by the VATS technique using robotic assisted thoracic surgery (RATS).

\section{The da Vinci ${ }^{\circledR}$ surgical system}

The dVS consists of a surgeon's console connected to the body of the dVS, a manipulator unit with 3-4 instrument arms, including a central arm to guide the endoscope camera, to which the surgeon's movements are transmitted, shown in Figure 1.

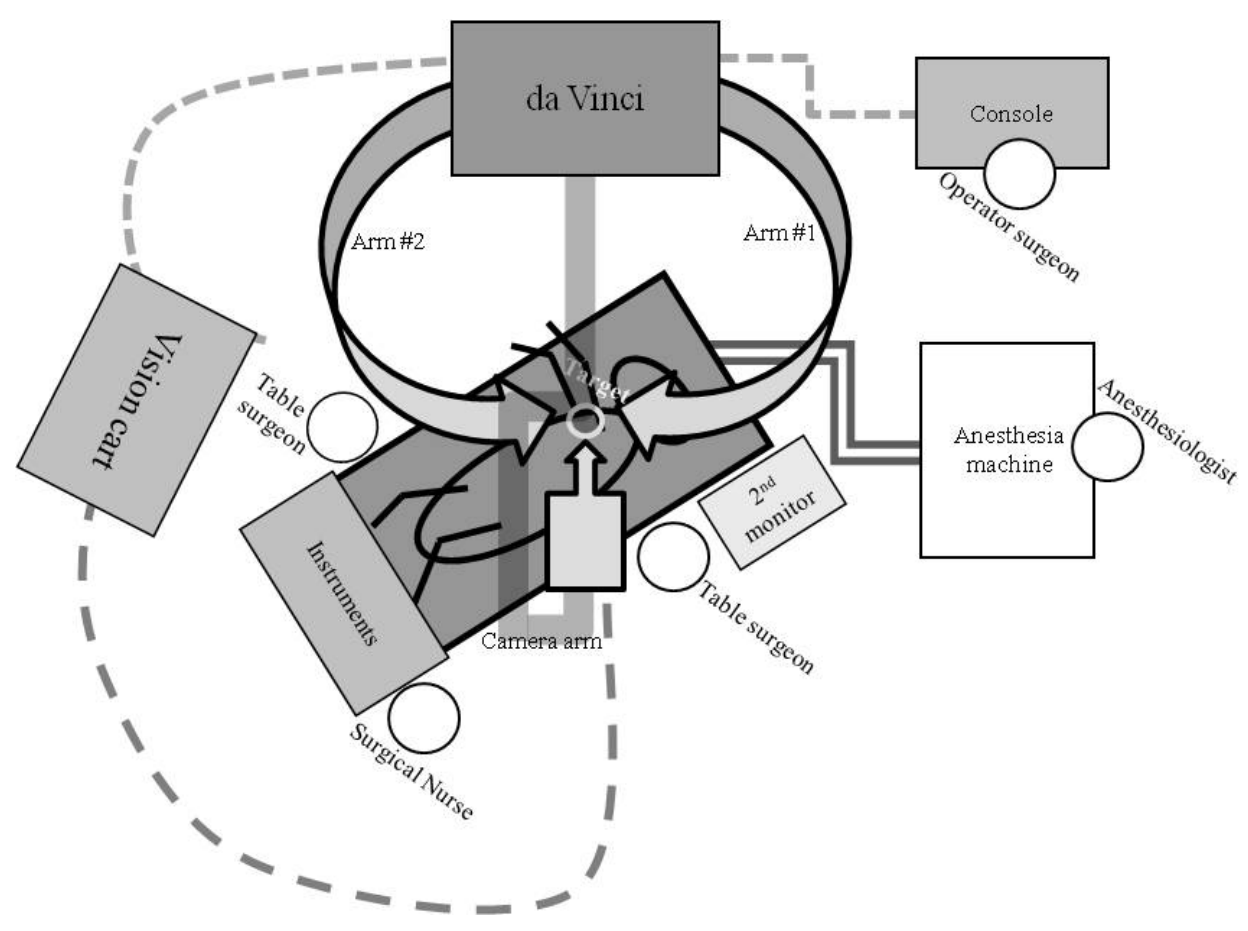

Fig.1-a 


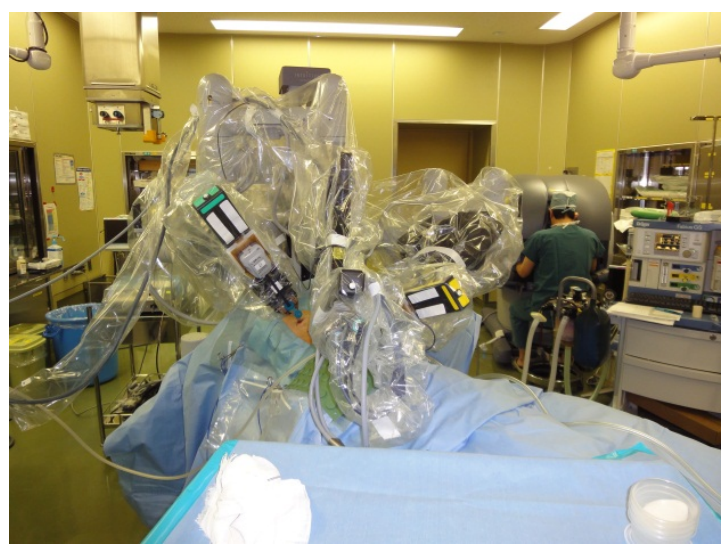

Fig. 1-b

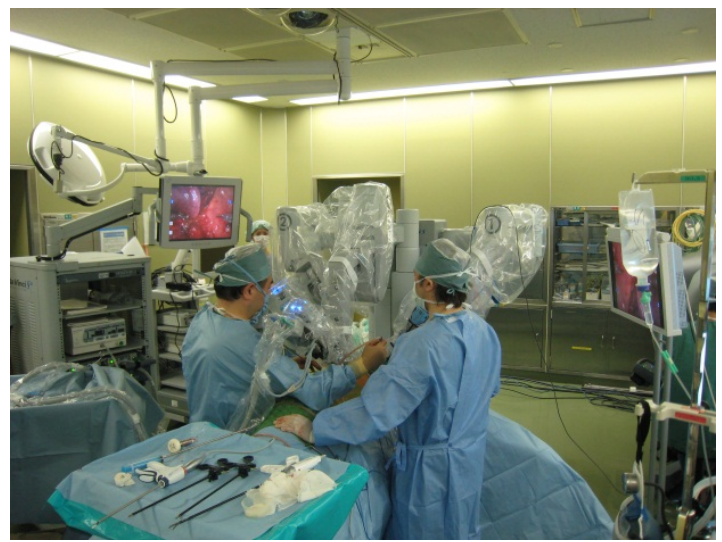

Fig.1-c

Figure 1. Positioning of all units for RATS

Appropriate positioning of all units for RATS is essential (Figure 1-a). The operating surgeon sits at the console (Figure 1-b) and there are two operating table surgeons (Figure 1c), an anesthesiologist and a surgical nurse. The roles of table surgeons include setting and adjustment of the arms of the dVS, changing of instruments, surgical assistance using VATS devices and, most importantly, conversion to open thoracotomy if necessary, e.g. in cases of massive hemorrage, during robotic surgery.

Accurate setup of the dVS is crucial to the success of thoracic operations, and this varies according to the location of the target in the mediastinum [4].

The flexible joint movements of robotic surgery enables dissection of targets located in sites that are difficult to access in the thoracic space, such as the upper, lower, anterior and posterior mediastinum. Dissection of various type mediastinal tumors with this system 
enables an accurate, smooth, and safe surgical operation, because the range of motion of the robotic arms and wrists within small spaces is extremely extensive. The EndoWrist ${ }^{\circledR}$ operative arm of the dVS is capable of replicating minute human wrist-like movements within the thoracic cavity (shown in Figure 2). Moreover the EndoWrist ${ }^{\circledR}$ system has motion scaling that eliminates physiological vibrations of the hands of the human surgeon.
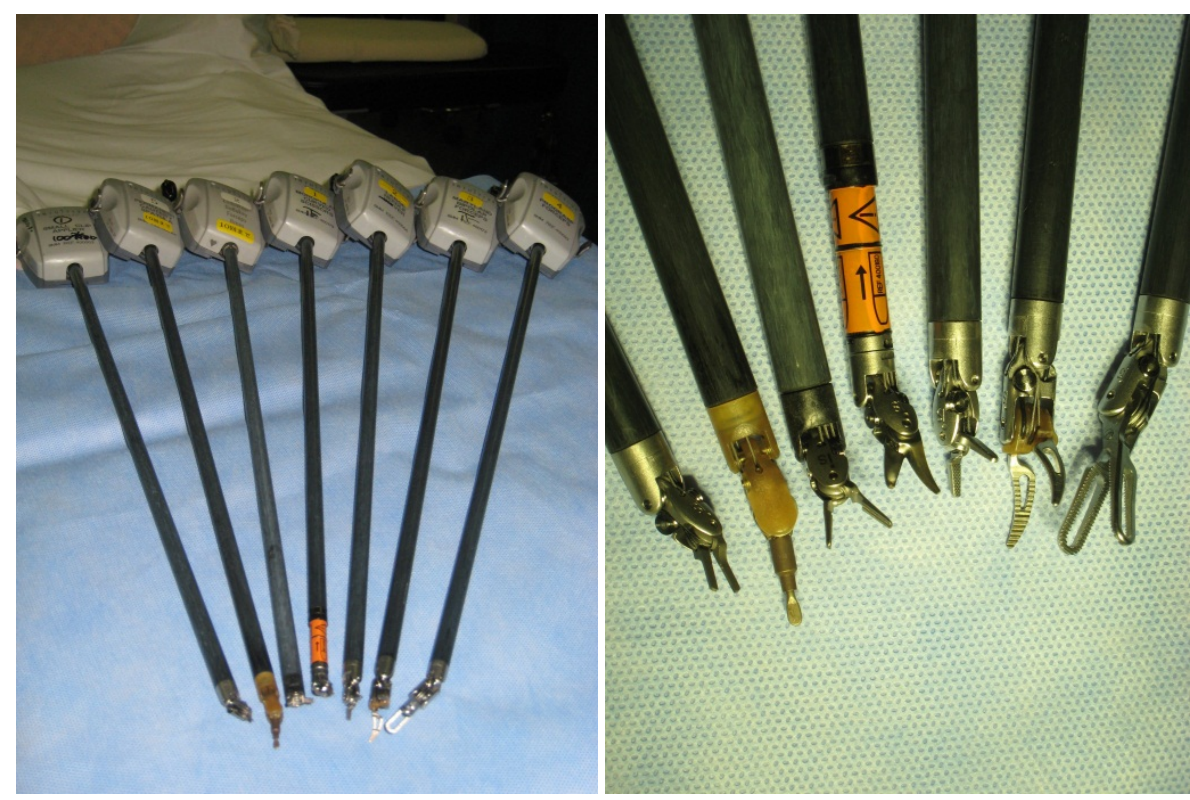

Figure 2. Instruments device

Figure 2 shows some of the accessory arms which are useful for general thoracic surgery (from left side; small clip applier, permanent cautery spatula, DeBakey forceps, monopolar curved scissors, large needle driver, Maryland bipolar forceps, ProGrasp forceps; usually attached the monopolar curved scissors on arm 1; as the right hand in thoracic procedure, and Maryland bipolar forceps or Cadiere forceps on arm 2; as the left hand in thoracic procedure).

The endoscope of the dVS system provides a 3-dimensional high-resolution binocular view of the surgical field, and is capable of a 12-fold enlarged view. The operation with the system enables accurate and simple safe surgery, because the range of motion of the robot arms within small spaces such as the thorax is extremely extensive. The crucial factors for successful dVS surgical procedures in thoracic surgery are selection of the appropriate placement and angle of the dVS ports, selected in relation to the individual target and patient position, which varies according to the target location. 


\subsection{Robot assisted thoracic surgery (RATS)}

\subsubsection{RATS set up}

Care in selecting the pre-setting according to target location is the most important stage for robotic surgery in such lesions. Adjustments to the position of the instrument arms are also necessary, depending on the level of the target lesion.

Typical positioning of the instrument arm ports shown in Figure 3 is important. The camera port is placed approximately $20 \mathrm{~cm}$ from the target through the disposable 12-mm trocar. Instrument arm ports are placed at least $10 \mathrm{~cm}$ away from the camera port or the other Intuitive instrument arm ports. Assistant port (s) consisting of disposable 5-12 $\mathrm{mm}$ trocars are placed at least $5 \mathrm{~cm}$ away from any camera or instrument arm ports.

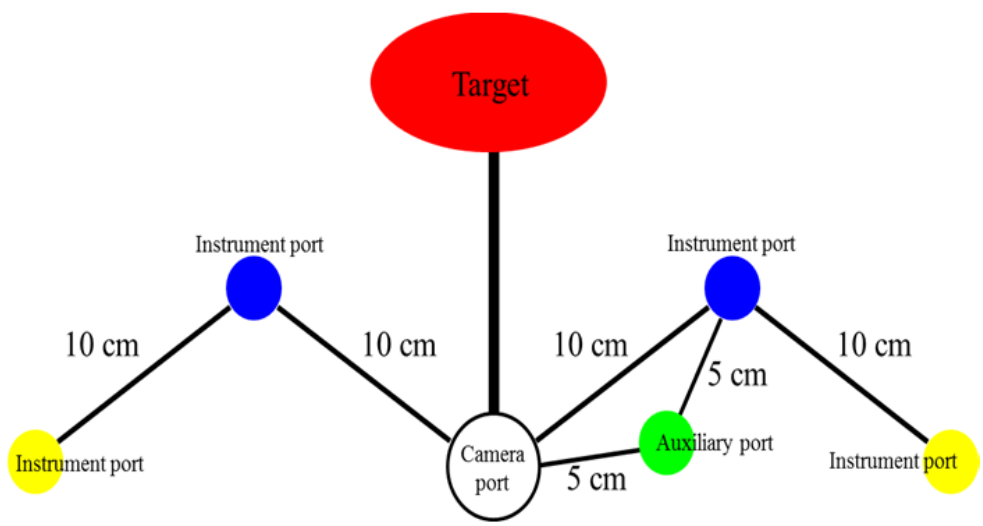

Figure 3. Positioning of Instrument Arm Port

- In the instrument-port procedure, the first port is positioned for the 3-dimensional camera placed in the 6-7 $7^{\text {th }}$ intercostal space on the mid-axillary line as shown in Figure 4.

- Another two ports for arms \#1 (right hand in the thoracic procedure) and \#2 (left hand in the thoracic procedure) are made. Arm port \#1 is usually placed in the $6-8^{\text {th }}$ intercostal space on the mid-posterior axillary line, and arm port \#2 is often placed in the $4-6^{\text {th }}$ intercostals spaces on the anterior axillary line for left-sided anterior tumors, as shown in Figure 4.

- $\quad$ The $4^{\text {th }}$ port is used as an accessory port for other devices such as those for vessel sealing and clipping, continuous suction, and washing. It is placed at least $5 \mathrm{~cm}$ apart from the other ports to prevent clashing of instrument arms. This accessory port is usually placed at the additional $1-2 \mathrm{~cm}$ incision made in the anterior axillary line in the $7-8^{\text {th }}$ intercostal space.

- $\quad$ The settings of the dVS for thoracic surgery are generally classified into four patterns. The placement of arm-ports for typically lobectomy and thymectomy are classified in the pattern of Figure 4, and as for posterior targets, the approach shown in Figure 5 is recommended [5]. 
- For targets located in the opposite side of the thoracic cavity, the system and instrument ports positions are reversed, and areas for arms \#1 and \#2 should be changed correspondingly.

- The patient position on the operating table is also extremely important for the dVS procedure. A semi-lateral prone position is appropriate for resection of posterior targets, to avoid contacting the lung anteriorly under gravity. The lower limbs of the patient are also flexed downward so that the pelvis does not disturb the movement of the instrument arms [5].

- In urgent conversion to open thoracotomy cases, it is important to mark a line on the skin between the instrument-ports of arm $\# 1$ and arm $\# 2$.

- To widen the narrow working space, CO2 inflation into the thoracic space (high-flow; $8-10 \mathrm{~mm} \mathrm{Hg}$ ) is carried out during the dVS procedure.

As Figures (4-6) show, target level is very important to decide the direction of approach of the dVS. Such this idea is a very crucial procedure to lose a perioperative blind spot in thoracic space.

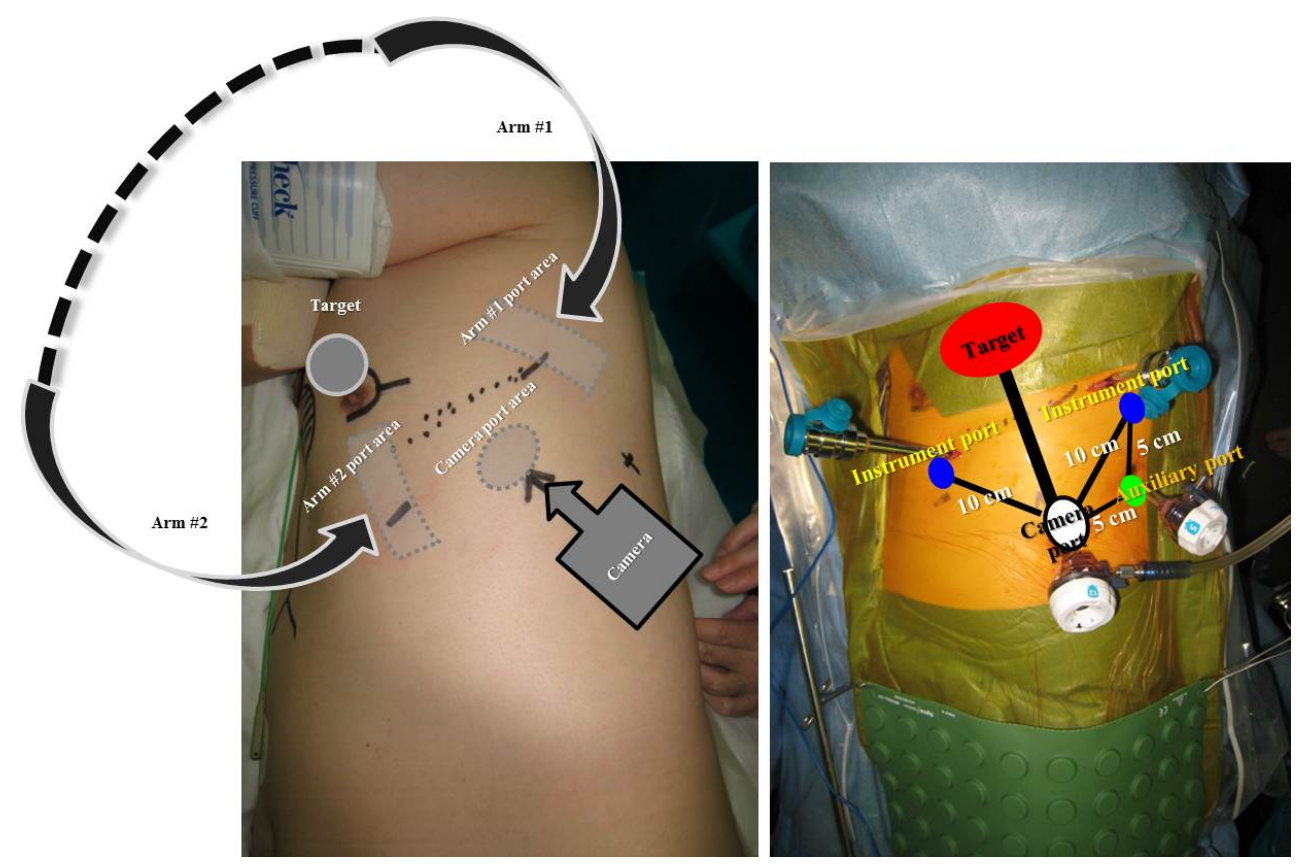

Figure 4. Appropriate parts replacement for thymectomy and lobectomy

The camera port is placed approximately $20 \mathrm{~cm}$ from the target through a disposable 12-mm trocar. Instrument arm ports are placed a minimum of $10 \mathrm{~cm}$ away from the camera port or the other Intuitive instrument arm ports. The assistant port, containing disposable 5- to 12$\mathrm{mm}$ trocars, are placed at least $5 \mathrm{~cm}$ away from any camera or Intuitive instrument arm ports. Instrument arms are very useful in general thoracic surgery, especially the monopolar 
curved scissors, the Maryland bipolar forceps, Cadiere forceps and the DeBakey forceps and the permanent cautery spatula.
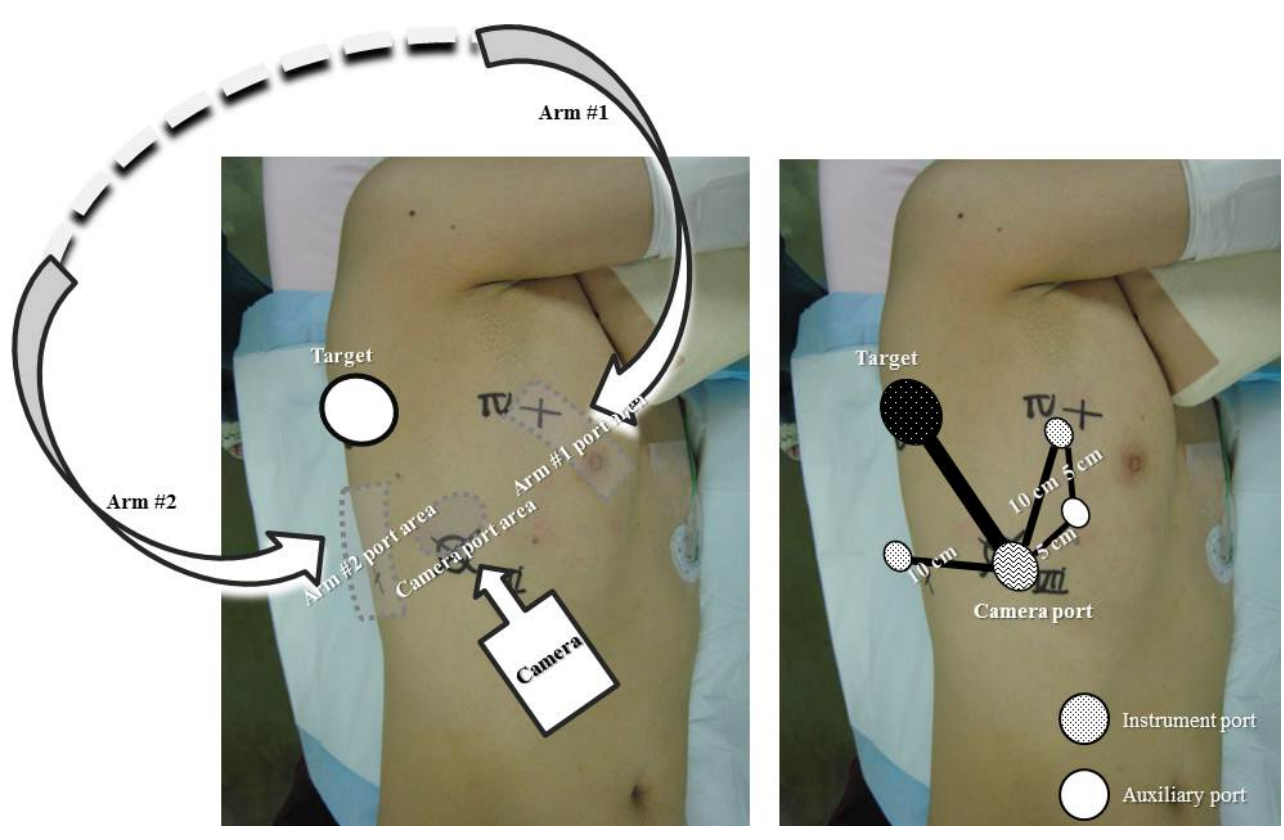

Figure 5. Appropriate placement of instrument ports for posterior tumors

Figure 5 shows the pattern of the location for right posterior-upper target with appropriate placement of instrument arm ports. The 3-D camera port is placed in the area of $6^{\text {th }}$ intercostal in anterior-mid axillary line. Arm \#1 is placed in the $3-4^{\text {th }}$ intercostal in anterior axillary line. Arm $\# 2$ is placed in the $8-9^{\text {th }}$ intercostal in the mid-posterior axillary line. The accessory port is placed in the $5^{\text {th }}$ intercostal space in the anterior axillary line. 


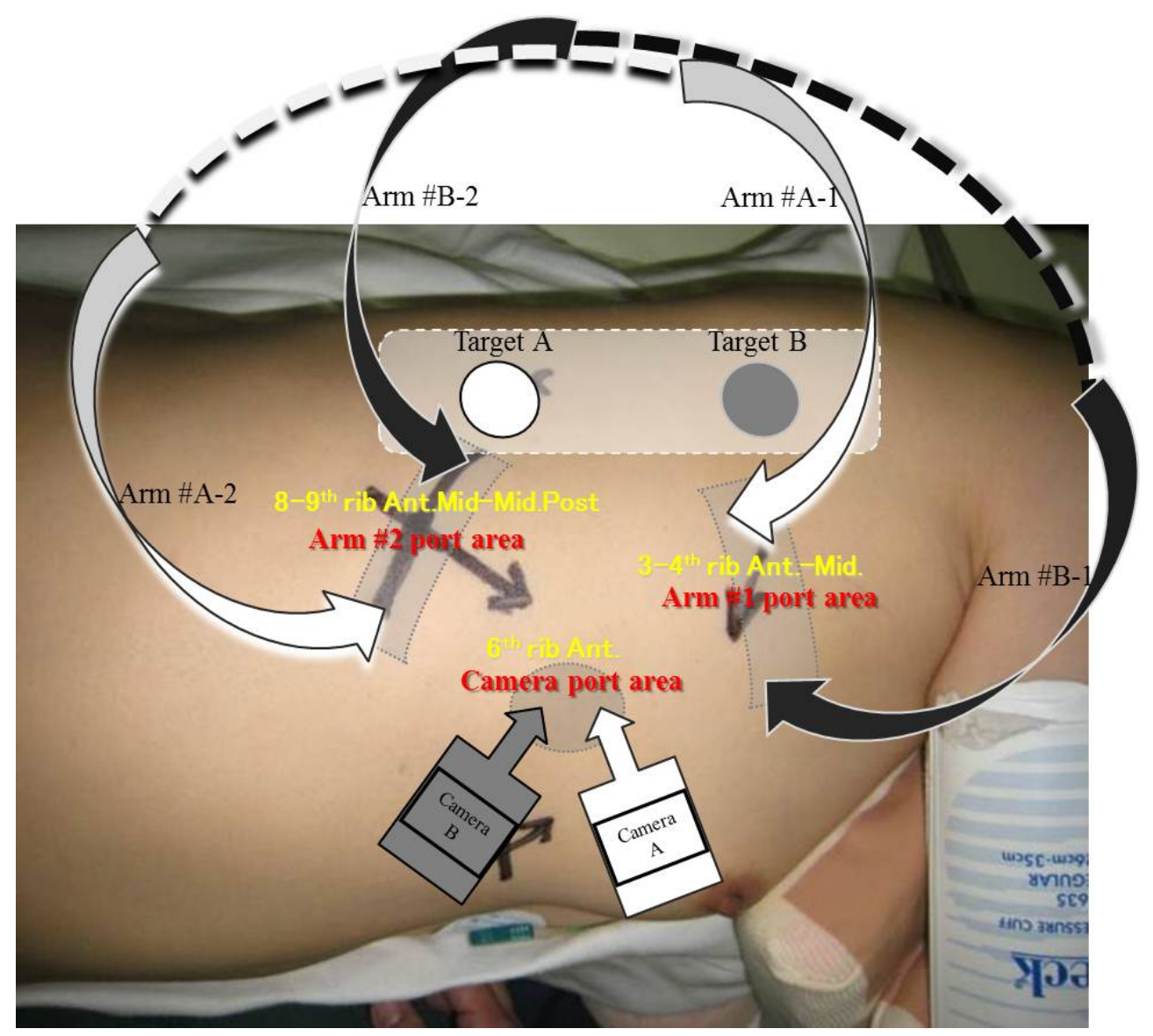

Figure 6. Appropriate placement of instrument arm ports for the right posterior targets location 


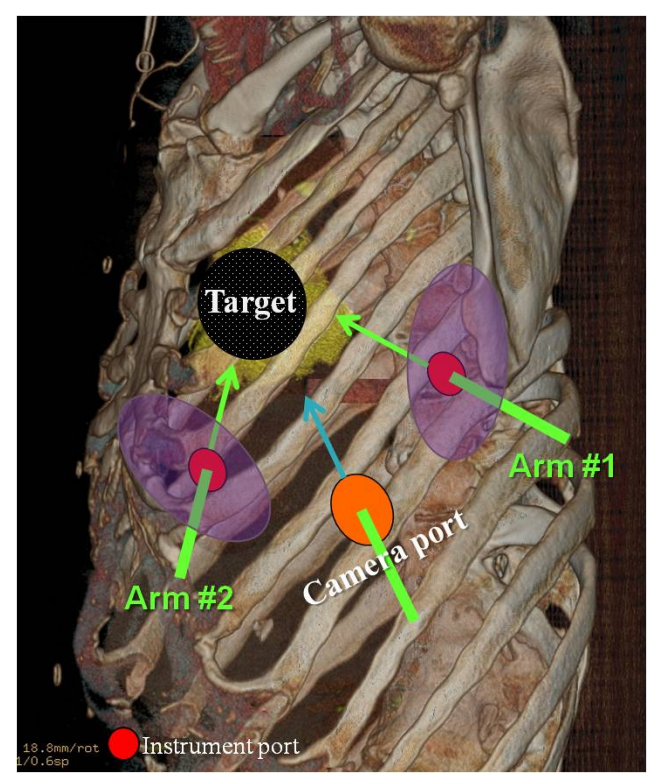

Fig. 7-a

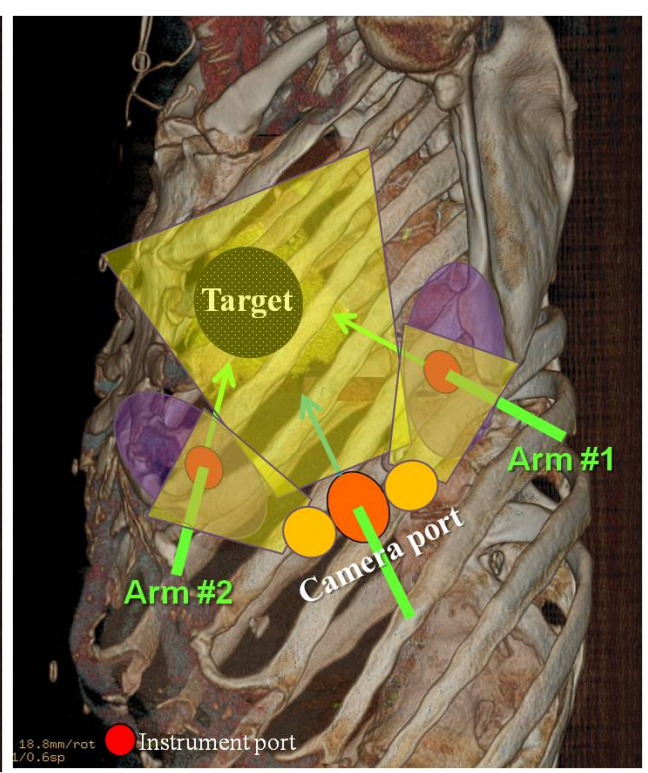

Fig. 7-b

Figure 7. Pitfalls of RATS 
Target level is important to decide the direction of approach of the dVS. Instrument arm ports for a right posterior target: the camera port is placed in the $6^{\text {th }}$ intercostal space area on the anterior axillary line; arm \#1 (A and B) is placed in the $3^{\text {rd }}-4^{\text {th }}$ intercostal space in the anterior axillary line; arm \#2 (A and B) is placed in the 8-9 $9^{\text {th }}$ intercostal space in the midaxillary line; an accessory port is placed in the $5^{\text {th }}$ intercostal space in the anterior axillary line. For a left-sided target, both arms ( $\# 1$ and \#2) should be interchanged.

\subsubsection{Pitfalls of RATS}

Arms of the dVS can be difficult to change position intraoperatively unlike in VATS so that preoperative fixation is important as described previously. That is the reason why the primary positioning of each unit and setting of instrument arms of the dVS is an essential step for the RATS procedure. The most important point regarding safety is to ensure a setting that eliminates or minimizes any blind spots of the console-operator, depending on target localization.

In clinical cases, trouble due to inappropriate manipulation in the blind spots (Figure 7-a; violet areas) in thoracic procedures seem empirically to depend on lack of function in that the tentacle of the arm tip against the organ is not transmitted to the console-operator. If the instruments give damage to lung in blind area, the operator sitting in the console could not know how to avoid this accident except for confirming with their own eyes.

In insertion of devices into the thoracic cavity and also in replacing them, it is essential during the process to confirm the exact position of the arm tip while watching the movement of the arms in the thoracic cavity closely by camera surveillance. Furthermore, table surgeons occasionally need to confirm the intrathoracic space with another submonitor or wide-view camera for safe manipulations during operation shown in Figure 7-b. These pitfalls are also crucial points for procedures in the abdominal space, and steps to avoid them can lead to the minimization of accidents.

Violet areas, which areas show the intrathoracic instrument port, are sometimes blind-spot from central camera view.

\section{Future developments}

RATS is generally better for the treatment of targets located in difficult to access areas than conventional VATS. However, the positioning of all units and the location of arm ports need suitable directional setting, which depends on the target location.

Several support safety developments are expected in the near future.

These ideas include following;

- instruments with tactile sensor tips,

- wide-angle view combined with the 3D main camera to eliminate blind spots,

- installing several supplementary cameras for total coverage of blind areas, 
- devices uses in conventional VATS, i.e., stapler, vessel sealing system, soft coagulation device.

Reduction in size and weight, and a more economical price is always a problem for the dVS.

In addition to reduction in size, the manipulation of several arms through a single thoracic incision has become possible. Furthermore, surgeons should be able to perform detailed treatment within the thoracic space even in areas difficult to reach and very tiny spaces in which to perform manipulation. In this way possibilities will continue to improve. Moreover the speed of such improvements might begin to increase exponentially.

That this is technology for the patient entirely is something that a surgeon must never forget, and it means that primarily we must think first about safety for patients.

\section{Author details}

Naohiro Kajiwara*, Masatoshi Kakihana, Jitsuo Usuda, Tatsuo Ohira, Norihiko Kawate and Norihiko Ikeda

Department of Surgery, Tokyo Medical University, Japan

Naohiro Kajiwara* and Norihiko Kawate

Department of Health Science and Social Welfare, Waseda University School of Human Sciences, Japan

\section{Acknowledgement}

The authors are grateful to Prof. J. Patrick Barron, Chairman of the Department of International Medical Communications of Tokyo Medical University for reviewing the English manuscript.

\section{References}

[1] Kajiwara N, Taira M, Yoshida K et al. (2011). Early experience using the da Vinci ${ }^{\circledR}$ surgical system for the treatment of mediastinal tumors. Gen Thorac Cardiovasc Surg. 59: 693-698

[2] Rückert JC, Swierzy M, Ismail M. (2011). Comparison of robotic and nonrobotic thoracoscopic thymectomy. A cohort study. J Thorac Cardiovasc Surg. 141(3): 673-7.

[3] Park BJ, Melfi F, Mussi A, Maisonneuve P, Spaggiari L, Da Silva RK, Veronesi G. (2012). Robotic lobectomy for non-small cell lung cancer (NSCLC): Long-term oncologic results. J Thorac Cardiovasc Surg. 143(2): 383-9.

[4] Kajiwara N, Kakihana M, Kawate N et al. (2011). Appropriate set-up of the da Vinci ${ }^{\circledast}$ surgical system in relation to the location of anterior and middle mediastinal tumors. Interact Cardiovasc Thorac Surg. 12: 112-116

\footnotetext{
${ }^{*}$ Corresponding Author
} 
[5] Kajiwara N, Kakihana M, Usuda J et al. (2012). Extended indications for robotic surgery for posterior mediastinal tumors. Asian Cardiovascular \& Thoracic Annals. 20(3): 308 313 